Katarzyna Karpińska-Szaj

Bernadeta Wojciechowska

Uniwersytet im. Adama Mickiewicza w Poznaniu kataszaj@amu.edu.pl,bewoj@amu.edu.pl

\title{
LA PERFORMANCE MORPHOSYNTAXIQUE DANS LES TÂCHES DE REFORMULATION ÉCRITE. CAS D'ÉTUDIANTS DÉBUTANTS DE FLE
}

Morphosyntactic performance in written reformulation tasks.

A case of beginners learning French as a foreign language

In this article the relation between the discourse production dynamic and the linguistic competence development is discussed. This problem is considered with regard to two types of language activities based on reformulation tasks: 1) written reconstruction of a story heard, 2) written production of the end of the story. The analysis of experimental data shows that in the second case the approach to the discourse is more complete which positively influences application of complex morphosyntactic structures in students production.

Keywords: teaching/learning French as a foreign language, morphosyntactic subsystem, reformulation, learning strategies, discourse competence

Słowa kluczowe: nauczanie/uczenie się języka francuskiego jako języka obcego, podsystem morfosyntaktyczny, przeformułowanie, strategie uczenia się, kompetencja dyskursywna

\section{Introduction}

L'enseignement de la grammaire dont la maîtrise devrait garantir à l'apprenant d'une langue étrangère la communication conforme au «bon usage» ${ }^{1}$,

\footnotetext{
${ }^{1}$ L'évolution du français (et d'ailleurs de chaque langue) prouve que ce qui est considéré comme écart à la norme peut changer de statut et entrer dans l'usage. Dans le
} 
a connu différents sorts. Après une période d'écartement de l'enseignement explicite du sous-système morphosyntaxique, les règles de bon usage sont réapparues dans les pratiques de classe, néanmoins leur présentation était soumise à des acceptions linguistiques différentes: grammaire notionnelle/fonctionnelle, grammaire énonciative, grammaire de sens, pragmatique linguistique, approche actionnelle, etc. L'optique discursive, laquelle est aujourd'hui incontournable dans les démarches d'enseignement d'une langue étrangère, et en l'occurrence de son sous-système morphosyntaxique, constitue pour nous aussi une plateforme de réflexion et de pratiques en classe de FLE. C'est dans ce cadre qu'il s'agira de comprendre le traitement de données morphosyntaxiques et de données discursives, et surtout leur mise en commun dans le parcours personnel d'apprentissage.

L'objectif de cet article n'est pas de nier les acquis positifs de l'enseignement explicite de la grammaire, mais de s'interroger plutôt sur l'intérêt pédagogique de ce déplacement d'accent vers les paramètres de discours susceptibles d'orienter la performance grammaticale de l'apprenant. Les effets qu'une telle insertion de l'enseignement de la grammaire dans le cadre discursif peuvent induire sur le processus d'apprentissage (et donc sur la construction/appropriation du sous-système morphosyntaxique), nous semblent particulièrement importants. La prise en compte de la norme discursive et de la règle grammaticale conjointement nous intéressera ici comme vecteur de l'autonomie de l'apprenant. Or, dans l'optique ici décrite, l'autonomie concernerait le développement de capacités d'observer et de produire des formes morphosyntaxiques dans le respect de la norme discursive et en rapport avec les intentions de communication sous-jacentes.

Le problème de l'articulation entre la dynamique de la production du discours et la dynamique de la construction de la compétence linguistique esquissé ci-dessus sera abordé dans notre article par rapport à la situation d'apprentissage du FLE au niveau débutant. II sera envisagé dans deux types d'activités langagières se basant sur les tâches de reformulation: une restitution écrite d'un récit auditionné et la production d'une suite d'histoire. Comment la part grammaticale et la part discursive (p.ex. connaissance des paramètres situationnels, respect du schéma narratif) de la compétence de communication se combinent-elles dans les deux situations d'apprentissage? La capacité de détecter les paramètres du discours par rapport à l'agencement du texte,

contexte d'enseignement/apprentissage du FLE, il devrait pourtant être envisageable de penser la norme (et, par conséquent, le bon usage conçu dans le sens grevissien comme ensemble de règles partagées par la société) en fonction de sécurité de l'apprenant qui a besoin de se référer à des modèles fiables. 
peut-elle devenir une stratégie de compensation de déficits de compréhension? Quels profits d'apprentissage peut-on tirer de ces deux tâches?

Ces questions vont nous conduire à débattre les points suivants: la prise en compte des paramètres discursifs en tant que facteurs favorisant (parfois même conditionnant) l'usage des structures morphosyntaxiques, la posture d'apprentissage (le vouloir progresser en langue) comme vecteur attentionnel lors de tâches de reformulation, et enfin, les conséquences du contrôle personnel des processus de la mise en forme sur l'apprentissage de la grammaire. Nous allons illustrer notre discussion sur ces problèmes de données expérimentales recueillies auprès d'étudiants débutants de philologie romane.

\section{Intégrer les données morphosyntaxiques et discursives: défis didactiques}

Comme nous l'avons déjà signalé dans l'introduction, l'apprentissage des structures morphosyntaxiques ne saurait être envisagé seulement à partir d'un traitement isolé des phénomènes linguistiques. Certes, il devrait se faire à travers la recherche et l'intégration d'une règle syntaxique insensible aux variations contextuelles mais aussi, parallèlement, à la mise en forme grammaticale, à travers la reconstruction de normes discursives présidant à son usage dans diverses situations de communication. On aura donc d'une part, des objectifs relatifs à la correction formelle, et d'autre part, des objectifs relatifs à la congruité discursive - adéquation par rapport au contexte (type et genre de discours) dans lequel les structures peuvent être utilisées en tant que porteuses de sens. II en résulte que les structures syntaxiques ne pourront pas être considérées comme pleinement acquises si elles sont envisagées par l'apprenant comme neutres sur le plan des pratiques sociales et le sens qui en découle. Bien au contraire, elles devraient, à notre sens être acquises comme indexées à un style, un type de discours et/ou à un genre.

Du point de vue des démarches d'enseignement, autant ces premiers objectifs relatifs à la règle, de par l'unicité et invariabilité de celle-ci, sont relativement faciles à formuler, autant les seconds objectifs sont plus problématiques en raison de l'hétérogénéité des normes de discours dans lesquels une structure prend son sens. Pour cette raison, ainsi que pour des raisons d'autonomie des étudiants adultes (devant être capables d'enrichir de façon autonome leur répertoire syntaxique au contact des productions discursives diverses), on retient l'hypothèse que ce qui est capital dans la formation d'étudiants c'est la mise en place d'une sensibilité aux régularités formelles en rapport à un contexte plus large dans lequel elles apparaissent. Par cet aspect, la notion de genre pourrait s'avérer de grande utilité en ce qu'elle explique probablement le mieux l'existence des normes, des scenarios intériorisés auxquels les interlocuteurs 
se réfèrent plus ou moins consciemment lors de la planification et de la réalisation des leurs énoncés en production et orientant l'écoute et l'interprétation en réception (Wilczyńska et Wojciechowska, sous presse). On pourrait considérer, en effet, le genre comme une instruction relative à l'intégration des niveaux linguistique, formel et de contenu ${ }^{2}$. La forme peut être donc désormais comprise de façon plus large comme englobant non seulement les moyens langagiers mais aussi la macrostructure du discours.

Les études sur la gestion du travail hypothético-référentiel lors de la compréhension orale et écrite (par exemple Cordier et Gaonac'h, 2010) ont démontré qu'une aisance élevée au moins d'une de ces catégories de connaissances peut favoriser le traitement global du discours en facilitant la sélection des éléments importants, en limitant le nombre d'inférences nécessaires en compréhension et en facilitant l'intégration des données en compréhension et en production. La familiarité avec le contenu d'un texte et/ou la bonne connaissance d'une macrostructure (dans notre expérience, du schéma narratif) peuvent même atténuer les effets d'une compétence linguistique limitée en langue étrangère et même servir de pistes de conceptualisation de structures grammaticales.

Il est pourtant évident que la réalisation des tâches impliquant l'intégration des niveaux linguistique, formel et de contenu est dotée d'une charge cognitive qui diffère par rapport au niveau d'habileté de l'apprenant quant à la forme, au référent et à la maîtrise de la compétence linguistique. Pour apprendre à gérer mieux ces va-et-vient entre différentes ressources mentales lors de la compréhension et de la production langagière, et en l'occurrence, pour développer les compétences morphosyntaxiques intégrées à la compétence discursive, il nous semble intéressant d'exploiter le modèle de sensibilité langagière. Dans ce modèle, la sensibilité est fondée sur la capacité de gérer l'attention, avant, durant et après l'activité de communication envisagée comme une situation d'apprentissage et elle est comprise comme un facteur dynamisant le développement des compétences (voir dans Wojciechowska, 2007). Le modèle de sensibilité langagière s'inspire des travaux menés dans le courant de SLA qui ont permis de formuler un certain nombre de conditions permettant à l'apprenant d'activer et de soutenir son apprentissage à partir des données disponibles dans l'environnement discursif en langue étrangère. Elle tient compte également des travaux sur l'attention, et en particulier ceux de Tomlin et Vila (1994) qui distinguent trois modes de fonctionnement de l'attention (dans Mialet, 1999), à savoir:

\footnotetext{
${ }^{2}$ Les bases de connaissances/compétences de l'apprenant se rapportant à la langue utilisée, au référent et à la forme, sont souvent abordées en termes de schéma. II est notamment question des connaissances et des représentations liées au schéma de contenu, au schéma linguistique et au schéma formel (voir p. ex. Denhière et Baudet, 1992).
} 
La performance morphosyntaxique dans les tâches de reformulation écrite ...

- la vigilance - une veille globale de l'esprit, la disponibilité à traiter des informations apparaissant dans l'environnement;

- l'orientation - une allocation des ressources de l'attention en direction des données spécifiques, qu'elle soit consciente ou non;

- la détection - l'enregistrement cognitif des données nouvelles sur lesquelles s'est focalisée l'attention.

Les études de Schmidt (par exemple, 1990) autour de noticing hypothesis montrent qu'une veille globale sur le discours en langue étrangère qui parvient à l'apprenant ne suffit pas pour que l'acquisition puisse avoir lieu. L'intégration d'un élément nouveau serait plutôt favorisée par la focalisation des ressources attentionnelles sur l'élément choisi, son enregistrement et sa compréhension. Ajoutons à cela que le repérage permettrait de toucher seulement à la surface d'un problème, à remarquer une occurrence d'un phénomène syntaxique (voir aussi Kasper et Rose, 2002: 21). Il aurait donc besoin de se poursuivre afin que l'apprenant puisse en saisir des règles syntaxiques en rapport avec des schémas discursifs plus généraux. Cela revient à envisager l'apprentissage non seulement comme un produit mais surtout comme un processus. D'autres résultats (par exemple, Swain, 1995) montrent que l'effort attentionnel est plus intense et plus efficace quand l'apprenant est amené à produire des structures nouvelles que quand il ne fait que les observer. La pertinence de cette proposition tient, entre autres, au fait, que comme l'a fait remarquer Carroll (dans Wong et Simard, 2001), I'intégration d'un nouvel élément ne s'opère pas à partir d'une production de l'autre mais à partir de la représentation que l'apprenant s'en fait ${ }^{3}$.

Cette optique du travail sur le contrôle des procédures morphosyntaxiques renvoie aussi à l'utilisation des stratégies, lesquelles prennent dans le contexte d'apprentissage une forme spécifique. Selon la classification de Béguin (2008) ${ }^{4}$, les stratégies impliquées dans la résolution de tâches sont relatives à l'anticipation et l'autorégulation. L'anticipation permet aux apprenants d'envisager les procédures nécessaires pour exécuter une tâche et, en même temps, pour satisfaire les objectifs d'apprentissage, tandis que l'autorégulation devrait garantir une meilleure adaptabilité à la situation donnée. Or, les activités de l'apprenant effectuées pour vérifier des contenus linguistiques

\footnotetext{
${ }^{3}$ Les travaux en question portent sur le repérage et l'apprentissage des éléments du système de la langue (lexique-grammaire), les processus touchant à la contextualisation, décontextualisation et recontextualisation de ses données restent toujours à éclairer.

${ }^{4}$ Il existe différentes classifications de stratégies d'apprentissage, nous utilisons dans cet article la répartition selon le critère d'implication métacognitive de l'apprenant pour planifier, résoudre et évaluer les problèmes d'apprentissage.
} 
travaillés en cours (par exemple: la recherche dans le texte entendu d'un élément de grammaire comme une justification de l'emploi des temps ou des articles régissant un point de vue, le prélèvement du vocabulaire) jouent sur la réception d'un texte entendu et par conséquent, sur la qualité de la reformulation. II s'agit ici du problème de distribution de l'attention sur les aspects propres à la tâche qui varient par rapport aux contraintes internes (déroulement du discours et son traitement, réussite ou échecs de traitements intermédiaires) et des contraintes externes (objectif de la tâche, compréhension de la consigne, conditions environnementales de l'activité en jeu, satisfaction des intérêts d'ordre plus général que la réalisation de la tâche). Dans ce sens, la sensibilité langagière est de prime importance dans le comportement stratégique de l'apprenant qui cherche à émettre des hypothèses sur le contenu et la forme tout en les modulant lors de la compréhension ou de la production langagière.

Dans le contexte d'apprentissage d'une langue étrangère, il est donc question de développer la capacité de coordonner le travail sur les tâches de

reformulation sur deux plans: celui de compréhension/interprétation et de l'exploitation des items dans la tâche suivante (la reformulation et la production d'une suite d'histoire) et celui du traitement de ces activités à des fins d'approfondissement des compétences linguistiques. Le défi pédagogique consiste à soutenir des outils cognitifs et métacognitifs que l'apprenant découvre lui-même avec, si nécessaire, le soutien ajusté de la part de l'enseignant. En effet, l'élève construit ses connaissances/compétences dans ses interactions avec l'enseignant ou/et avec ses pairs par les interventions de nature métalinguistique et métacognitive. La conceptualisation de la langue devrait donc être étayée par des activités de recul métalinguistique à des fins de généralisation et de renforcement de la structuration des connaissances (voir aussi Chini, 2010). L'objectif didactique majeur est donc de soutenir l'effort de l'apprenant pour qu'il puisse connaître, réguler et autoévaluer ses propres démarches cognitives en vue de réaliser des buts personnels d'apprentissage par l'intermédiaire des tâches proposées.

\section{La performance morphosyntaxique lors des tâches de reformulation: données expérimentales}

\subsection{Le cadre expérimental}

Afin d'analyser les rapports entre les données discursives, la performance morphosyntaxique et les objectifs personnels d'apprentissage, nous avons adapté le protocole de recherche du projet Acquisition de la complexité linguistique de 
6 à $14 a^{a n s^{5}}$. Ce projet a pour but d'expliquer comment l'enfant transforme la langue utilisée dans son entourage (ceci est démontré à partir des procédures de reformulation utilisées pour restituer le texte source lu par l'expérimentateur) pour construire par la suite son énoncé. Suivant le but de cet article, nous avons décidé de voir si et dans quelle mesure les tâches de reformulation d'un récit peuvent être exploitées par nos étudiants débutants dans leur réflexion sur l'apprentissage/usage de la grammaire dans l'optique discursive. La capacité à reformuler le texte source afin de rapporter son sens original relève de la capacité à comprendre le texte auditionné et de la capacité à produire un texte reformulé tout en gardant dans l'esprit la constante sémantique globale (le sens du texte), c'est-à-dire, en gardant constante la conceptualisation du contenu à narrer (cf. Karpińska-Szaj et Paprocka-Piotrowska, 2014). De cette façon, le texte reformulé serait la verbalisation de cette conceptualisation et, par conséquent, la trace observable de la performance morphosyntaxique utilisée pour reconstruire un récit discursivement organisé.

Les étudiants de la deuxième année de philologie romane à l'Université de Poznań (8 personnes débutantes, niveau A2) ont été priés d'effectuer deux tâches:

- restituer avec leurs propres mots une histoire lue par l'expérimentateur (voir dans l'annexe) et, une semaine après,

- écrire la suite de cette histoire (la longueur maximale: 1 page).

Ces tâches ont chacune les caractéristiques propres. La première peut être considérée comme beaucoup plus contraignante puisque la restitution du sens de l'histoire suppose le respect des étapes de l'histoire et de leur enchaînement, ce qui limite la possibilité de manœuvre aussi sur le plan grammatical. En même temps, le texte auquel l'apprenant est exposé lui fournit un cadre discursif bien défini et un inventaire d'outils grammaticaux sur lesquels il peut s'appuyer pour réaliser l'activité. La deuxième tâche est beaucoup plus libre, elle n'impose que le début de l'histoire et donne libre voie à l'imagination (le sens) que l'apprenant doit pourtant organiser selon un schéma de son choix et en fonction des moyens langagiers à sa disposition.

Tandis que dans la première tâche il était intéressant de voir si et dans quelle mesure les données discursives s'emboîtent à l'usage de la grammaire par rapport au texte source, dans la deuxième tâche, il était question d'analyser à cet égard les productions libres (non reformulées du texte source). L'enjeu

\footnotetext{
${ }^{5}$ Le projet est coordonné par prof. Claire Martinot (Paris-Sorbonne) et regroupe des chercheurs de France, Italie, Pologne, Croatie et Allemagne travaillant dans le cadre de l'équipe Sens Texte Informatique Histoire (EA 4509 STIH). L'équipe polonaise est représentée par Urszula Paprocka-Piotrowska (Université Catholique Jean Paul II, Lublin); Katarzyna Karpińska-Szaj et Bernadeta Wojciechowska (Université Adam Mickiewicz, Poznań).
} 
didactique consistait à les comparer selon leur complexité morphosyntaxique et lexicale envisagée par rapport à la complétude discursive. Or, conformément à l'objectif de notre expérience (examen de la performance morphosyntaxique par rapport aux caractéristiques discursives du récit présenté), nous avons discerné pour l'analyse de la première tâche, les paraphrases formelles et explicatives produites par les étudiants. Les paraphrases formelles résultent de la transformation de la construction phrastique: le même lexique est repris dans une construction différente, p. ex. passif vs actif, permutation d'un constituant, nominalisation d'un verbe, etc. (cf. Martinot, 2015). Dans les textes des étudiants, elles peuvent donc traduire le niveau de la performance morphosyntaxique (les reformulations, sont-elles erronées? sont-elles conditionnées par la compréhension du récit?). Par contre, selon la définition donnée par Martinot (ibidem), les paraphrases explicatives sont définies comme relatives au discours et, lors de l'acquisition de la langue maternelle, elles «ne présentent pas de contraintes formelles, en revanche, elles introduisent un nouveau point de vue, une cause, une conséquence ou une justification. Elles nécessitent une grande maitrise textuelle, et/ou une connaissance du monde et des contraintes pragmatiques (...)».

Les connaissances générales de l'apprenant semblent parfois guider le choix (bien que celui-ci soit relativement limité) des constructions verbales. Dans notre expérience, ce type de paraphrase permet donc de révéler:

- la qualité de la compréhension des événements de l'histoire,

- la superposition du schéma narratif supposé connu des étudiants en tant que facilitateur de la compréhension et, par conséquent, de l'organisation du texte reformulé.

Il convient de souligner aussi que la réalisation de tâches de reformulation peut être utilisée en termes de stratégies de conceptualisation des structures morphosyntaxiques à condition d'accompagner le travail sur la reformulation $d^{\prime}$ 'une réflexion sur la nature et les conditions d'usage de la grammaire. Ceci dit, les pratiques stratégiques se basant sur la reformulation devraient déboucher sur 'l'éclairage' du cheminement mental de l'apprenant amenant à la production des contenus observés pour que celui-ci puisse les réutiliser de manière consciente dans d'autres situations d'apprentissage. Pour cette raison, les tâches de reformulation ont été suivies d'entretiens individuels qui avaient pour but d'inviter les étudiants à la prise de conscience des procédures d'autocontrôle effectuées.

\subsection{La présentation et discussion des résultats}

Avant de commencer l'analyse, disons seulement que la qualification du groupe d'étudiants dont les productions sont prises en compte dans notre recherche 
La performance morphosyntaxique dans les tâches de reformulation écrite ...

comme relevant du niveau $\mathrm{A} 2$ ne veut pas dire que le niveau des étudiants particuliers est le même. Les différences interindividuelles sont parfois très importantes. Nous avons pourtant réussi à dégager certaines tendances qui sont récurrentes dans toutes les copies et c'est essentiellement sur ces éléments que nous voulons insister.

\subsubsection{Les paraphrases formelles dans la tâche de reformulation contrainte par l'écoute}

Ainsi, la première observation qui s'impose après la lecture des restitutions d'étudiants dans la tâche de reformulation contrainte par l'écoute porte sur la simplifixification syntaxique et lexicale par rapport au texte auditionné. Le corpus analysé contient très peu de paraphrases formelles qui témoigneraient d'une focalisation sur la structure morphosyntaxique des énoncés. Nous illustrons ce constat à partir de la séquence 13 de l'histoire qui comporte un pronom interrogatif au discours indirect, réputé difficile à contrôler par les apprenants au niveau A2.

Comme on le voit dans cette séquence le pronom «ce qui» est trois fois utilisé dans le même cotexte: "Tom a répondu: "Je veux apprendre à parler avec les oiseaux qui savent tout ce qui se passe dans le ciel, avec les poissons qui savent tout ce qui se passe dans l'eau et avec les fourmis qui savent tout ce qui se passe sur la terre». On pourrait s'attendre à ce que cette triple répétition retienne l'attention des apprenants et se traduise par une reformulation correcte de ce passage. L'analyse des résultats cependant révèle que la majorité des apprenants restitue l'idée de communication avec des animaux comme source de connaissance sans s'inspirer du patern morpho-syntaxique, ce qui donne des énoncés comme:

(1) AM: «Il a voulu parler avec l'oiseau et l'autre animaux pour savoir beaucoup des choses qui se passent dans la Terre».

Il y a deux apprenants qui restituent la structure de la phrase de départ AK et $\mathrm{JK}$, mais I'un d'eux la remplit avec des pronoms incorrects:

(2) JK: «Tom a demander de pouvoir comprendre les oiseaux qui savent qu'est qu'il passe au l'air, les poissons qui savent qu'est qu'il passe à l'eau et les fourmis qui savent qu'est-ce qu'on passe dans la terre».

Le seul qui restitue les pronoms relatifs correctement est AK, mais il commet de nombreuses erreurs à d'autres endroits, comme on peut le voir dans le passage ci-dessous: 
(3) AK: «Le garçon a voulu de connaitre le mots les oiseau pour savoir ce qui se passe sur le ciel, le mot de poissons pour savoir ce qui se passe dans l'eau et les mots de fourmis pour savoir ce qui se passe sur la terre».

Parallèlement, l'analyse des productions dans la tâche 2 a montré que la plupart des personnes utilisent des propositions complétives introduites par le pronom interrogatif correctement. Citons à titre d'exemple TP.

(4) TP: «Tom était surpris parce qu'il comprenait tout ce qu'un oiseau disait» ou un peu plus loin chez le même apprenant «un oiseau a raconté tout ce qu'il voulait savoir...».

\subsubsection{La complexité morphosyntaxique envisagée par rapport à la complétude discursive}

Sur le plan discursif, dans la première tâche on peut noter la focalisation des apprenants sur ce que l'on pourrait considérer comme éléments obligatoires du récit. En plus, les actions principales, indispensables pour restituer le sens de l'histoire et sa cohérence ont été reformulées par les apprenants de manière synthétique, ou autrement dit sans décomposition en une suite d'actions «mineures», constitutives en quelque sorte de l'action principale.

Observons cette tendance en référence à la séquence 3: Le lendemain matin, dans la cour de l'école, Tom guettait l'arrivée de sa nouvelle petite voisine. Dès qu'il l'a aperçue, il s'est dirigé vers la fillette et lui a tendu la boîte qu'il avait fabriquée pour elle, la veille. Dans cette séquence, l'acte principal d'offrir le cadeau est décrit dans une suite d'actes mineurs exprimés par les verbes guetter, apercevoir, se diriger et tendre. Un tel procédé stylistique permet de créer un effet de tension qui suscite l'intérêt de l'auditeur/lecteur et lui facilite l'imagination de la situation racontée. Or, dans les restitutions des apprenants ces procédés discursifs sont très rares. Ce qui domine en revanche, ce sont des phrases courtes, simples, où l'action principale est présentée, on pourrait dire de manière concentrée. En voilà quelques exemples:

(5) JK: «Tom a donné le cadeau pour Julie. C'était la boîte.»

(6) PT: «... il a fait une boîte de couleur rouge et l'a donné à elle.»

(7) AM: «Le jour aprés pendant le cour il a donné ce cadeau à Julie.»

Dans les énoncés d'apprenants, la complexité se limite à l'utilisation des compléments $\mathrm{COD}$ et $\mathrm{COI}$ qui pourtant sont moins nombreux que dans le texte de 
La performance morphosyntaxique dans les tâches de reformulation écrite ...

départ. On note peu d'opérations syntaxiques et grammaticales complexes comme la subordination ou la concordance des temps ${ }^{6}$.

La focalisation sur les éléments obligatoires se fait au détriment des éléments facultatifs concernant le décor de l'action et les sentiments des protagonistes. Les observations relatives aux éléments facultatifs du schéma narratif peuvent être exemplifiées en référence à la séquence 12 , comportant une courte description du jardin: Tom et Julie se trouvaient dans un jardin merveilleux où les fleurs semblaient se parler en chantant. Alors Julie dit à Tom: "Viens, traversons le jardin, il y a une grande fête pour toi, ce soir. Jusqu'à minuit, tu as le droit de demander à notre Roi tout ce que tu veux».

Dans la tâche contrainte par l'écoute, pas une seule occurrence de description du jardin n'a été trouvée, sur six copies dans deux seulement le jardin apparaît mais sans aucun qualificatif ni développement sur l'apparence de cet endroit. Voilà les deux prédications:

(8) AK. Soudain les enfants étaient dans le jardin.

(9) AM: Ils ont entrés dans le jardin.

La description de la forêt ou du jardin dans la tâche 2 non seulement apparaît mais elle donne lieu à une complexité syntaxique importante avec le recours à des propositions subordonnées (dans la plupart des cas, complétives ou relatives):

(10) PT «Et soudain il s'est trouvé dans un endroit magnifique. C'était le forêt où il y avait pleins d'oiseaux.»

(11) AK: «Il pouvait appercevoir la mer au gauche et un grand rouge lac à droite. Dans le ciel il avait plein des oiseaux qui chantaient et qui volaient entre les arbres».

(12) KK: «C'était le meilleur lieu dans le monde. C'était la nature qu'il n’a jamais connu avant».

Les apprenants détaillent mieux, dans cette deuxième tâche, les actions du protagoniste ce qui contribue à construire la dynamique de la narration. Les exemples illustrant cette tendance sont nombreux, en voilà quelques-uns:

(13) JK: «Lentement il a regardé à ce qui était à l'autre côté et il a tourné sa tête et son visage tout blanc pour s'assurer que Julie y était.»

(14) AM: «et puis il avait l'impression qu'ils ne sont pas tout seul. Les oiseaux sont venu chez lui, ils se sont aproché et ils ont commencé chanter pour lui».

\footnotetext{
${ }^{6}$ Notons au passage que la recherche a été effectuée après une période de travail sur l'emploi et la concordance des temps.
} 
En effet, les exemples présentés ci-dessus tout en illustrant une plus grande complexité syntaxique révèlent aussi une plus grande prise en compte du point de vue du protagoniste - de ses émotions, de ses sentiments, de ses motifs. En voici quelques exemples encore:

(15) RG: «Heureusement apres quelques jours il a appris leur langage parce que tout les oiseaux était très gentils et adorables».

(16) JK: «En ouvrant la porte Tom était stressé.»

L'analyse a mis en évidence un plus grand emploi des paternes syntaxiques complexes (gérondif, adverbes, propositions relatives, explicatives, causales, etc. impliquant souvent la concordance des temps) dans la deuxième tâche mais elle a aussi montré que les apprenants ont encore du mal à surveiller les formes verbales, les prépositions, l'orthographe, bref, les éléments du niveau le plus bas. Malgré ces quelques difficultés de correction, au niveau discursif, leurs productions apparaissent comme beaucoup plus complètes. On peut penser que c'est cette posture de narration, plus visible dans la deuxième tâche, qui entraîne l'emploi des structures plus complexes, nécessaires pour mettre en rapport les actions et les sentiments qui les accompagnent ou les justifient.

\subsubsection{L’impact des représentations des apprenants sur la réalisation des tâches}

Les entretiens que nous avons menés et enregistrés avec les apprenants juste après la réalisation de la deuxième tâche, et donc une semaine après la réalisation de la première tâche, apportent certaines explications aux résultats obtenus. Nous avons orienté nos entretiens de façon à obtenir des informations sur la gestion des ressources cognitives dans les deux tâches. Nous avons fait I'hypothèse que cette gestion dépend de la perception de l'objectif de chacune des tâches par rapport 1) au sens, 2) aux formes (discursives + morphosyntaxiques) et 3) à la manière dont l'apprenant l'inscrit dans ses visées d'apprentissage personnelles.

La majorité des étudiants a évalué la première tâche comme plus difficile à réaliser que la deuxième. Pour deux personnes la rédaction de la suite de l'histoire a été problématique non pour des raisons langagières mais parce qu'elles disaient avoir du mal à «inventer des histoires». Les étudiants interprètent la première tâche majoritairement comme une tâche de vérification de compréhension orale, ce qui a pour corolaire leur concentration sur le sens. Ils pointent de façon unanime les limites de leur mémoire qui rendaient la 
La performance morphosyntaxique dans les tâches de reformulation écrite ...

mémorisation de formes linguistiques difficiles. JK explique le processus en question comme suit: «J'avais un problème avec l'écoute à savoir que dans la tête cela se transposait en polonais et je mémorisais le sens en polonais et après ces mots qui apparaissaient et que je comprenais en raison du contexte, je ne pouvais pas les réutiliser de nouveau en français parce que je les connaissais seulement de ce contexte et non dans la traduction du polonais au français» ${ }^{7}$.

Une étudiante, AK, se distingue de ce point de vue des autres, elle dit s'être intéressée aussi bien aux formes qu'au sens, il lui paraissait évident que si «L'histoire se déroulait dans le passé donc les temps étaient importants»": Elle dit même éprouver une sorte de satisfaction à reconnaître les formes verbales récemment connues et à observer leur usage dans I'histoire puisqu'elle se rend compte du fait que ces formes récemment travaillées ne sont pas encore complètement acquises. D'après ces déclarations, on peut constater que ses stratégies d'écoute prennent également en compte le schéma narratif - dès le début, cette histoire renvoie, pour elle, à des histoires connues sur les enfants à l'école avec les étapes propres à ce genre de situations. Elle s'en sert pour soutenir sa mémoire «ce qui se passait dans l'ordre, [...] la maîtresse n'arrive pas, elle est en retard, arrive la nouvelle camarade, elle doit s'asseoir quelque part tout ça je le mettais dans I'ordre dans ma tête, comme un plan d'action ${ }^{9}$.

A part cet apprenant, les autres disent en général que la forme discursive n'attirait pas tellement leur attention. En même temps, contrairement à leurs déclarations pourrait-on dire, ils arrivaient, à notre demande, à en analyser la structure en se référant plus ou moins implicitement au schéma narratif et en associant les parties de I'histoire à différentes variantes du genre (histoire réaliste, fantastique). A la lumière de ces analyses, on peut avancer que tous les apprenants connaissent plus ou moins le schéma narratif, mais ils l'utilisent peu consciemment comme une stratégie d'écoute orientant leur attention sur les éléments de I'histoire et les structures morphosyntaxiques sous-tendues. Le choix de donner la priorité au sens pouvait influencer ainsi

7 "Z tym słuchaniem miałam taki problem, że w głowie przechodziło mi to na język polski i zapamiętywałam to po polsku i potem te słowa, które gdzieś się pojawiały i które zrozumiałam ze względu na kontekst, nie mogłam potem użyć ich z powrotem po francusku bo znałam je jakby tylko z tego kontekstu, a nie w tłumaczeniu z polskiego na francuski”. [JK, interview]

8 „Historyjka działa się w przeszłości dlatego ważny był czas." [JK, interview]

9 "Co się po kolei działo przychodzi pani znaczy nie przychodzi pani, spóźnia się, przychodzi nowa dziewczynka tutaj musi usiąść, to wszystko po kolei sobie w głowie układałam, jakby plan wydarzeń". [AK, interview] 
la concentration sur les événements racontés au détriment de leur inscription dans le contexte externe et interne de l'univers des protagonistes.

Pour ce qui est de la deuxième tâche, les apprenants la conçoivent comme plus axée sur les formes discursives et morphosyntaxiques mais aussi moins contraignante et plus confortable parce que permettant de gérer plus facilement les ressources langagières, le choix du vocabulaire et la construction de phrases. Ainsi, MK déclare: «quand j'écrivais mon histoire je pouvais écrire ce que je voulais, quand j'écoutais il fallait traiter ce que vous dites» ${ }^{10}$. JK approfondit la même idée en disant: «J'avais moins de problèmes avec la recherche de mots quand j'écrivais mon histoire, c'était comme si je connaissais mes limites, mes possibilités au niveau du vocabulaire donc j'essayais de ne pas les dépasser c'est probalement pour cela que je n'avais pas tellement de problème de vocabulaire ${ }^{11}$.

Malgré le sentiment d'insatisfaction exprimée face à la qualité de restitutions, les apprenants sont nombreux à dire que cette tâche précisément pourrait être utile pour leur apprentissage. Ils suggèrent que leur pratique régulière pourrait les aider à exercer leur mémoire et leur attention afin d'observer les formes en cours d'apprentissage et remarquer et retenir des formes nouvelles dans le discours de l'autre. Certains indiquent également la manière de raconter comme précieuse à observer - «j'apprends cette langue et je voudrais savoir raconter». Ils se rendent bien compte que les formes qu'ils apprennent actuellement ne sont pas pleinement opérationnelles et ils pensent que ce serait utile de les entendre dans différents contextes gérés par d'autres. Ils apprécient aussi la deuxième tâche dans la mesure où elle offre la possibilité de mettre en oeuvre ce qu'ils connaissent et l'approfondir dans un contexte nouveau géré par eux-mêmes.

\subsubsection{Conclusions}

La confrontation des réponses des étudiants avec leurs productions dans les deux tâches permet de constater que les conduites utilisées lors de la réalisation de la tâche de restitution ne sont pas les mêmes que celles utilisées lors de la tâche de reformulation libre. Dans ce deuxième cas, probablement en raison de la pratique d'enseignement de l'écrit à partir des genres et des types

10 "Gdy pisałam, mogłam pisać to na co mam ochotę, gdy słuchałam moim zadaniem było przetworzyć to co pani mówi." [MK, interview]

${ }^{11}$ Znacznie mniejszy problem miałam z szukaniem słów jak pisałam sama z siebie, to było tak jakbym znała swoje granice, możliwości swojego słownictwa więc starałam się nie uciekać za to pewnie dlatego ze słownictwem nie było takiego problemu. [JK, interview] 
La performance morphosyntaxique dans les tâches de reformulation écrite ...

de texte, la posture face au discours est plus complexe et plus complète, ce qui a permis aux apprenants d'intégrer dans la production du sens le niveau discursif et le niveau morphosyntaxique. C'est comme cela en tout cas que nous expliquons la complexité morphosyntaxique mais aussi une meilleure cohérence de l'histoire des productions de reformulation libre.

Ceci n'est pas vraiment le cas de productions contraintes par l'écoute où les données discursives ont été insuffisamment utilisées pour instruire les stratégies de compréhension. La posture d'écoute focalisée uniquement sur le sens ne permet pas de le saisir dans toute sa complexité et richesse parce qu'elle ne tient pas compte du cadre discursif justement et de la matérialité linguistique mise en oeuvre pour le construire.

Les deux tâches présentent selon les opinions des étudiants une importante valeur d'apprentissage puisqu'elles permettent de prendre conscience du parcours personnel (relatif aux expériences, à la diversité des compétences et aux objectifs d'apprentissage) de la (re)construction du sens lors de la restitution et lors de la production libre. Les différences retenues par les apprenants quant à la qualité des structures grammaticales utilisées dans les deux productions ont démontré que, contrairement à ce qu'ils ont pensé au départ, on ne peut pas les associer uniquement aux contraintes morphosyntaxiques (donc relatives à leur niveau de compétence en cette matière) mais également aux contraintes discursives et à la gestion de celles-ci dans la réalisation de tâches.

\section{Remarques terminales}

Dans cet article, nous avons examiné la rentabilité didactique des tâches de reformulation dans le développement de la performance morphosyntaxique des étudiants débutants. Les résultats de notre expérience ont montré que l'apprentissage de la morphosyntaxe intégré au discours pourrait se faire à travers l'accomplissement de tâches de reformulation qui exigent la mise en œuvre de plusieurs opérations mentales.

La première tâche, contrainte par l'écoute passait par: compréhension/ interprétation > mise en mémoire > conceptualisation en vue de production > décisions quant aux contenu, forme et moyens linguistiques pour produire un texte reformulé. Tous ces processus dynamiques et complexes en même temps, exigeaient de l'apprenant un grand éveil attentionnel qui bloquait parfois leur disponibilité langagière pour réaliser cette activité. La deuxième tâche qui consistait en la production d'une suite d'histoire, procurait aux étudiants plus de liberté (et de temps) quant aux possibilités d'étayer leurs ressources langagières des connaissances discursives. 
L'explicitation de la gestion de cette dynamique dans les deux tâches lors des entretiens avec les étudiants a permis d'entrevoir le fonctionnement du soussystème morphosyntaxique dans sa dimension sociale mais aussi personnelle qui englobe la spécificité de «pratiquer la langue» dans la situation d'apprentissage.

\section{BIBLIOGRAPHIE}

Béguin, Ch. 2008. «Les stratégies d'apprentissage: un cadre de référence simplifié». Revue des sciences de l'éducation, vol. 34/1: 47-67.

Chini, D. 2010. «Langage et/ou action? La perspective actionnelle favorise-t-elle vraiment l'apprentissage linguistique?» In: Macaire, D., Narcy-Combes, J.-P. \& H. Portine (coord.). Interrogations épistémologiques en didactique des langues. Le français dans le monde. Recherche et application 48: 164-175.

Cordier, F. et D. Gaonac'h. 2010. Apprentissage et mémoire. Paris: Armand Colin.

Denhière, G. et S. Baudet. 1992. Lecture, compréhension de textes et sciences cognitives. Paris: Presses Universitaires de France.

Karpińska-Szaj, K. et U. Paprocka-Piotrowska. 2014. "La reformulation en tant que stratégie d'apprentissage d'une LE: vers son exploitation dans des situations non-ordinaires». Roczniki Humanistyczne LXII/10: 55-82.

Kasper et Rose, 2002. «Theories of Second Language Pragmatic Development». Language Learning Ajournal of Research in Language Studies. University of Michigan by Blackwell Publishing.

Martinot, C. 2015. "La reformulation: de la construction du sens à la construction des apprentissages». CORELA - cognition, représentation, langage. - sous presse.

Mialet, J.-P. 1999. L'Attention. Paris: PUF.

Schmidt, R. 1990. "The role of consciousness in second language learning». Applied Linguistics 11: 129-158.

Swain, M. 1995. "Three functions of output in second language learning». In G. Cook and B. Seidlhofer (éd.), Principle and practice in applied linguistics: Studies in honour of H.G. Widdowson. Oxford: Oxford University Press: 125-144.

Swales Woodfield, D. J. 1997. «The Language Teacher Output and Beyond to Dialogue: A Review of Merrill Swain's Current Approach to SLA». The Language Teacher - Issue 21.9.

http://www.jalt-publications.org/ttt/files/97/sep/woodfield.html consulté le 22 mai 2015.

Tomlin, R.S. et V. Vila. 1994. "Attention in cognitive science and second language acquisition». Studies in Second Language Acquisition, 16 (2), 183-203.

Wojciechowska, B. et W. Wilczyńska. (sous presse). «Procédés interactifs de construction du sens. Un modèle dans le développement des compétences orales élevées en langue étrangère?».

Wojciechowska, B. 2007. Le développement de la sensibilité langagière en communication orale exolingue au niveau avancé. thèse de doctorat non publiée.

Wong, W. et D. Simard. 2001. "La saisie, cette grande oubliée!», Acquisition et interaction en langue étrangère [en ligne], 14, mis en ligne le 15 décembre 2005. URL: http://aile.revues.org/1476 consulté le 22 mai 2015. 
Annexe: texte source (séquençage)

\section{Tom et Julie}

1) Ce matin-là, la maîtresse est arrivée dans la cour de l'école plus tard que d'habitude. Elle tenait par la main une petite fille que personne n'avait encore jamais vue.

2) Arrivée en classe, la maîtresse a dit: «Les enfants, je vous présente votre nouvelle camarade, elle s'appelle Julie. Tom, la place est libre à côté de toi. Julie sera ta voisine, sois bien gentil avec elle!»

3) Tom était fou de joie à l'idée d'avoir peut être une nouvelle amie. Le soir, chez lui, il a fabriqué une petite boîte ronde, rouge et dorée, pour Julie.

4) Le lendemain matin, dans la cour de l'école, Tom guettait l'arrivée de sa nouvelle petite voisine. Dès qu'il l'a aperçue, il s'est dirigé vers la fillette et lui a tendu la boîte qu'il avait fabriquée pour elle, la veille.

5) Julie aimait tellement cette boîte qu'elle la prenait toujours avec elle. Quand la maîtresse disait: «Sortez vos affaires!», Julie posait délicatement la boîte entre Tom et elle, sur leur table de travail.

6) Un jour, Julie chuchota à Tom: «Ouvre la boîte!» Tom souleva le couvercle et découvrit un morceau de papier sur lequel Julie avait écrit: «Je t’attends ce soir à 8h, sous le gros arbre, à l'entrée de la forêt».

7) Tom avait un peu peur parce qu'il lui était interdit d'aller dans la forêt, surtout la nuit.

8) Mais à $8 \mathrm{~h}$ du soir, il était tout de même au rendez-vous, Julie l'attendait déjà.

9) Sans dire un mot, la petite fille prit la main de Tom et frappa 3 fois sur le tronc du gros arbre.

10) Au bout de quelques minutes, les enfants entendirent un grincement. L'arbre était en train de tourner sur lui-même.

11) Tout à coup, le tronc s'ouvrit et les enfants furent éblouis par la lumière qui inondait l'intérieur de l'arbre. Ils firent quelques pas et l'arbre se referma derrière eux.

12) Tom et Julie se trouvaient dans un jardin merveilleux où les fleurs semblaient se parler en chantant. Alors Julie dit à Tom: «Viens, traversons le jardin, il y a une grande fête pour toi, ce soir. Jusqu'à minuit, tu as le droit de demander à notre Roi tout ce que tu veux».

13) Tom a répondu: «Je veux apprendre à parler avec les oiseaux qui savent tout ce qui se passe dans le ciel, avec les poissons qui savent tout ce qui se passe dans l'eau et avec les fourmis qui savent tout ce qui se passe sur la terre».

14) Et depuis ce jour, Tom est devenu un enfant extrêmement savant. 